\title{
Probe Shaping for Quantitative DPC-STEM Using Segmented Detectors
}

L. Clark $^{1}$, H.G. Brown ${ }^{1}$, D.M. Paganin ${ }^{1}$, M.J. Morgan ${ }^{1}$, T. Matsumoto ${ }^{2}$, N. Shibata ${ }^{2}$, T.C. Petersen ${ }^{1}$ and S.D. Findlay ${ }^{1}$

1. School of Physics and Astronomy, Monash University, Victoria, Australia

2. Institute of Engineering Innovation, University of Tokyo, Tokyo, Japan

Methods to measure phase-shifts imposed by samples in scanning transmission electron microscopy (STEM) are undergoing somewhat of a renaissance, following significant advances in fast pixelated electron detectors [1, 2, 3]. One particularly powerful method is MIDI-STEM [4], which combines a phase plate of Fresnel zones in the condenser aperture with correlated detection zones on a pixelated detector to produce essentially linear phase-contrast images, making for a robust and reliable technique. In this presentation, we explore how probe shaping can similarly generate a signal linear in the specimen phase gradient when used in conjunction with the more traditional quadrant-annulus segmented detectors.

One approach to differential phase contrast STEM (DPC-STEM) with segmented detectors is the rigid intensity-shift model, which assumes that the phase gradient of the specimen causes a rigid shift of the intensity profile in the detector plane (detectable as a difference in the signal strength on two diametrically opposed detector segments). Within this model, there are a number of assumptions that may be unmet in practice. The default probe in the STEM is an approximation to an Airy probe. Such probes have very broad, slowly decaying tail intensities, due to the sharp-edged aperture applied in the condenser plane, and thus sample regions of the specimen far from the central position of the probe. In interesting specimens, these regions may well encompass areas of varying phase gradient, preventing a true rigid intensity shift at the detector plane and instead introducing characteristic intensity peaks and troughs at the edges of the bright-field disc. With a standard segmented detector, we find two possible routes to circumvent this problem.

If the approximate specimen geometry can be assumed a priori (Fig. 1), then the lateral extent of the peak and trough features in the bright-field disc can be estimated (Fig. 2). It may then be possible to adjust the camera length and detector orientation such that these features are contained within a particular detector segment. The resultant measured centre-of mass, from a "rigid-shift" interpretation, is then close to that measured with a pixelated detector [5].

Alternatively, if the probe shape can be re-engineered such that the intensity of the probe tails decays more rapidly with radius, then the simple analysis method of the rigid intensity-shift model will become valid for a broader range of specimens. Recent developments into novel electron-optical setups have demonstrated that the condenser aperture can be varied rather freely $[6,7,8]$. The Gaussian probe phaseplate, recently demonstrated by McMorran et al. [9] holds great promise here - the Gaussian probe is more localised than an Airy probe of the same full-width half-maximum, and would thus be less prone to producing peaks and troughs in the bright-field disk region. Our initial simulations confirmed that an approximation to a Gaussian probe (formed by applying an aperture at the first intensity minimum of the Airy probe) is indeed expected to generate a highly accurate map of specimen phase gradients [10]. 


\section{References:}

[1] G McMullan et al., Ultramicroscopy 109 (2009), p.1126.

[2] MW Tate et al., Microscopy and Microanalysis 22 (2016), p.237.

[3] H Ryll et al., Journal of Instrumentation 11 (2016), 04006.

[4] C Ophus et al., Nature Communications 7 (2016) p.10719.

[5] L Clark et al., arXiv:1801.07572 (2018).

[6] J Verbeeck, H Tian and P Schattschneider 467 (2010), p.301.

[7] L Clark et al., Physical Review Letters 111 (2013), 064801.

[8] R Shiloh et al., Ultramicroscopy 144 (2014), p.26.

[9] BJ McMorran et al., FEMMS conference (2017), p.25.

[10] This research was supported by the Australian Research Council Discovery Projects funding scheme (Project DP160102338). N.S. acknowledges support from SENTAN, JST and JSPS KAKENHI Grant numbers JP26289234 and JP17H01316. The GaAs p-n junction samples were provided by Hirokazu Sasaki, Furukawa Electric Co., Ltd.

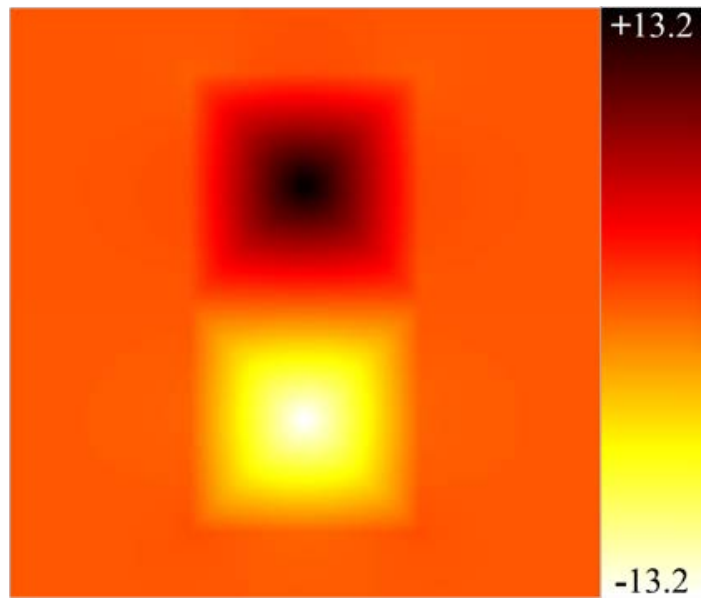

Figure 1. Phase map of sample magnetic domain model used in the study. Phase shifts vary between \pm 13.2 radians. Domain size is $1 \times 0.5 \mu \mathrm{m}$.

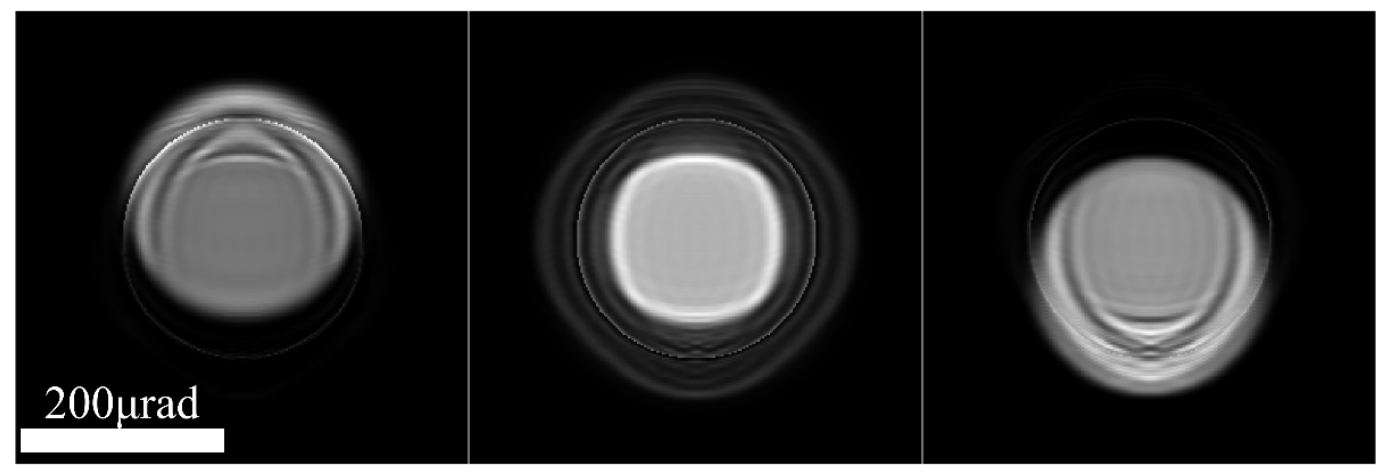

Figure 2. Sample ronchigrams from probe illuminating the phase map illustrated above. Clear deviations from rigid intensity shifts are visible. Simulations at $200 \mathrm{keV}$, convergence angle, $\alpha=133 \mu \mathrm{rad}$. 